\title{
ON THE INTERPRETATION OF SEISMIC CONE PENETRATION TEST (SCPT) RESULTS
}

\author{
IRENA BAGIŃSKA \\ Wrocław University of Technology, Faculty of Civil Engineering, Wrocław, Poland, \\ e-mail: irena.baginska@pwr.wroc.pl
}

WOJCIECH JANECKI

Geosoft, Ltd., Wrocław, Poland, e-mail: geosoft@geosoft.com.pl

MACIEJ SobÓTKA

Wrocław University of Technology, Faculty of Civil Engineering, Wrocław, Poland, e-mail: maciej.sobotka@pwr.wroc.pl

\begin{abstract}
The paper deals with the methodology of performing and interpretation of seismic cone penetration test (SCPT). This type of test is used to determine velocity of the seismic wave in the soil medium. This study is focused on shear wave. The wave is triggered on the ground surface by hitting an anvil with a sledgehammer. Then, vibrations induced at different depths are measured. Based on recorded measurements wave velocity $\left(V_{s}\right)$ and thus also small strain shear modulus $G_{\max }$ may be calculated. An interpretation of exemplary seismic test results is presented. Crossover and cross-correlation methods are discussed and another, more adequate one is featured and then applied in the interpretation example. Conditions for correct test performance and interpretation are discussed.
\end{abstract}

\section{INTRODUCTION}

Currently, commonly used CPTU probes are often equipped with additional measuring attachments to determine velocity of seismic waves. Two basic types of seismic waves can be distinguished: compression wave and shear wave. The former is referred to as the P-wave (primary wave) and the latter as the S-wave (secondary). Elastic constants $\left(E_{\max }, G_{\max }, v\right)$ can be calculated on the basis of determined wave velocities. The obtained moduli are called small strain, maximal or dynamic (Fajklewicz [4]). Elastic parameters derived are used in a wide range of analyses, especially associated with problems of foundation engineering.

The arrangement of the paper is the following. A methodology of in situ seismic test using a GEOTECH 220-04 CPTU penetrometer equipped with a seismic module is presented in the next section. Then the interpretation procedure with the use of SEISMIC-pro software is discussed and example results and their interpretation are presented. The conclusions are formulated at the end. 


\section{TESTING METHODOLOGY}

Seismic test (SCPT) consists in triggering a seismic wave in the soil medium by hitting an anvil resting on the ground surface with the use of a sledgehammer. Then, the wave is recorded by a system of accelerometers or geophones located behind piezocone. The vibrations registered for the vertical direction are called longitudinal, while those for the perpendicular (horizontal) direction are referred to as transverse. These types of vibrations are results of the P-wave and S-wave, respectively. The P-wave is a compression wave and S-wave is a shear wave. Measurements of the S-wave and/or P-wave are recorded at different depths, typically at every meter or every half a meter. The time it takes for the wave to reach the accelerometers at a given depth is evaluated. Differences of travel times at subsequent depth levels are determined. The time interval between two depths is determined. In this way wave velocities may be assessed and consequently, taking proper assumptions, other geotechnical parameters may be evaluated.

The seismic test can be performed simultaneously with a typical CPTU one. A standard CPTU test consists in pushing an instrumented probe, with the tip facing down, into the ground at a controlled speed rate $(2.0 \mathrm{~cm} / \mathrm{s})$. During the procedure four parameters are measured and registered each 1 second, i.e., every $2 \mathrm{~cm}$ of depth increase. The values being measured include cone resistance $\left(q_{c}\right)$, sleeve friction $\left(f_{s}\right)$, pore water pressure $\left(u_{2}\right)$, and inclination of the probe as a control parameter.

The additional seismic attachments for the CPTU probe include a seismic module synchronized with an electric conical tip, a cable for sending analog signals, an interface box, an anvil and a pedestal as well as a sledgehammer to induce seismic waves (Fig. 1).

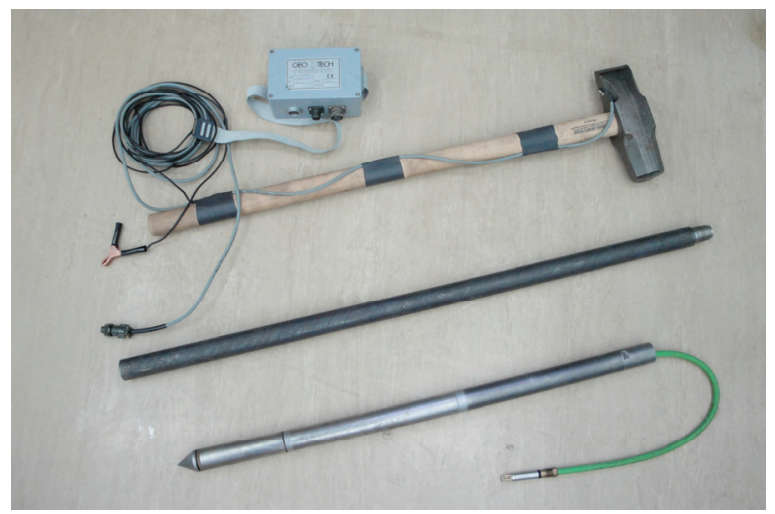

Fig. 1. Measurement equipment of the seismic module with single tri-axial accelerometer set

The seismic module is situated directly above the measurement cone in the CPTU probe. Inside the module there are three accelerometers arranged in three perpendicular directions. Two of them are horizontal $(X, Y)$ and one is vertical $(Z)$. During the measurement the accelerometers register vibrations resulting from a seismic wave induced on 
the ground surface level. An example of the seismic test results recorded from a single accelerometer, to be discussed later in the paper, is presented in Fig. 2. The test in which two signals are analyzed from two different depths by one geophone or accelerometer is called pseudo-interval and was used in surveys analyzed and published in the paper.

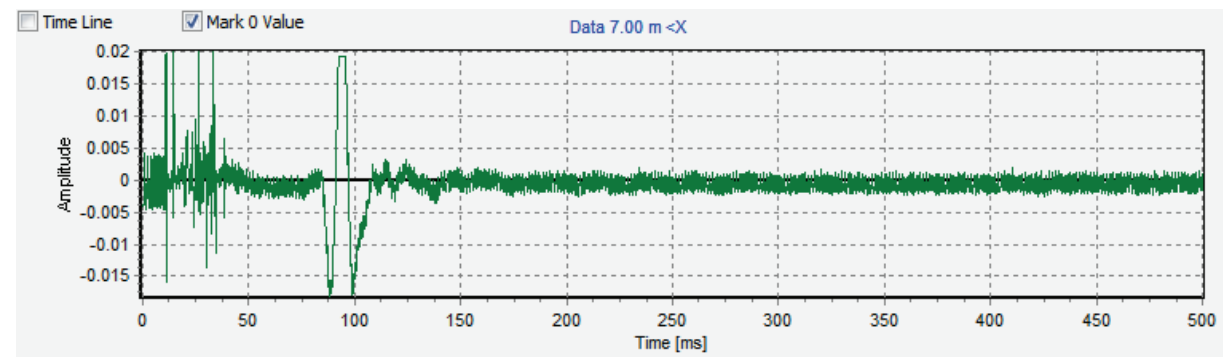

Fig. 2. An exemplary outcome of seismic test - a record of vibration in the $X$ direction

Measurements are typically taken at consecutive depths spaced at a distance of $1.0 \mathrm{~m}$. The S-wave is triggered twice for each particular measurement depth: on the left and then the right side of the rod. A scheme of this process is shown in Fig. 3.

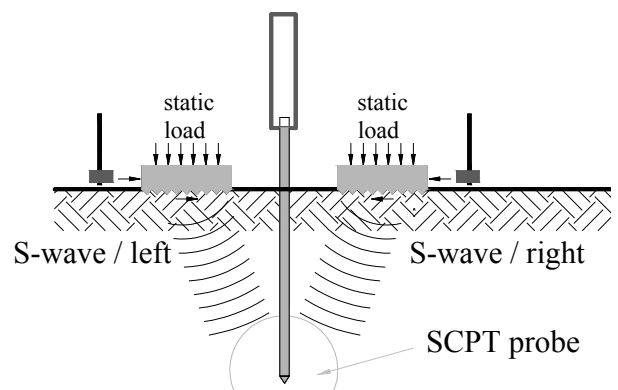

Fig. 3. Diagram of the S-wave measurement

The seismic wave is triggered by the hammer impact in the horizontal direction. The wave induces vibration of soil particles. The predominant direction of movement is horizontal, i.e., transverse to the vertical direction of wave propagation. Two identical anvils resting firmly on the ground are typically used. The anvils are set symmetrically to the rod. The wave is induced by the impact on the left and the right anvils for each measurement depth. An electrical circuit is closed by the contact of the hammer and the anvil when it is being hit. Then the wave is induced and the measurement record is being initiated at the same time. In every record there are indications for the three accelerometers, arranged in three perpendicular directions $(X, Y$ and $Z)$. The seismic test can be repeated at any depth. Adjusting the sensitivity range of the measuring instruments is advised to obtain the most readable results. 
The hammer strikes need to be performed with consistent intensity, paying careful attention to their "purity", that is a single contact from the hammer at each strike. Test quality significantly depends on the settlement of the anvil and its contact with the ground. The anvil should be properly connected to the ground and loaded. Vibrations affecting the pressing device should be avoided because they could disturb the measurement. These problems have already been addressed in literature on the subject (e.g., Bajda [3]). It should be remembered that the break for the seismic test should not be too long, because it can affect the conventional CPT test.

\section{INTERPRETATION OF SCPT RESULTS}

An exemplary seismic measurements, the interpretation of which is discussed in this section, was carried out simultaneously with a standard CPTU examination. The aim of measurements was to determine the geotechnical profile and the initial shear modulus of the subsoil. Plots of acceleration versus time recorded at different depths for the "left" and "right" waves are provided in Fig. 4.

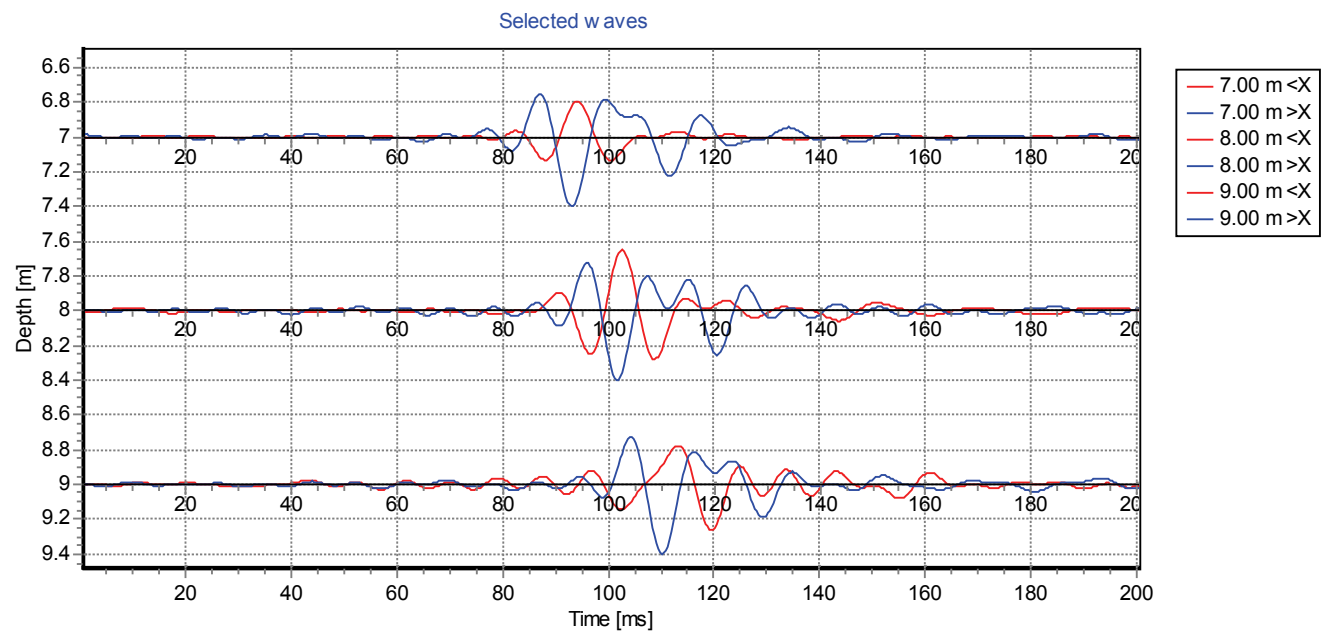

Fig. 4. "Left" and "right" wave records at depths of 7.0, 8.0 and $9.0 \mathrm{~m}$

The results are analysed in a two-step procedure. The first step involves determination of the time it takes for the wave to reach the sensors. Average velocity for a given depth interval is calculated on the basis of differences in arrival times measured at consecutive depths. This step was performed using SEISMIC-pro software. The results from the first step are incorporated in the second one, in which shear modulus is evaluated for given depth intervals. The second stage was conducted with the use of CPT-pro software. 


\subsection{DETERMINATION OF S-WAVE VELOCITY}

Measurements recorded for horizontal directions were analysed in order to determine velocity of the shear wave. Because more amplitudes were observed in the $X$ direction, the results for a single direction $(X)$ are discussed later in the article.

Calculation of the velocity is based on differences in time after which characteristic points of registered wave appear at plots referring to subsequent depths. The interval shear velocity is given by the distance interval divided by the time interval. Maximum of the "right" wave record and the corresponding minimum of the "left" wave record or vice versa, as well as zero points may be considered characteristic points of the plots. In another approach, the points where "left" and "right" graphs are crossed are investigated. Those methods are discussed in detail by Areias and Van Impe [1].

SEISMIC-pro software was used as an interpretation tool in the example discussed in this paper. The program is based on another method, which involves a comparison of wave records provided by striking a single anvil, either "left" or "right". An analysis taking into account a number of tests suggests that the "left" and "right" waves differ in more aspects than polarization only (see Fig. 4). The factors responsible for this fact include, e.g., inevitable differences in the way the anvils are attached, a lack of reproducibility of impulses and asymmetric behavior of accelerometers.

The method applied in SEISMIC-pro software is based on the following assumptions:

1. Single waves, either "left" or "right", are included in the determination of wave velocity for a given depth interval.

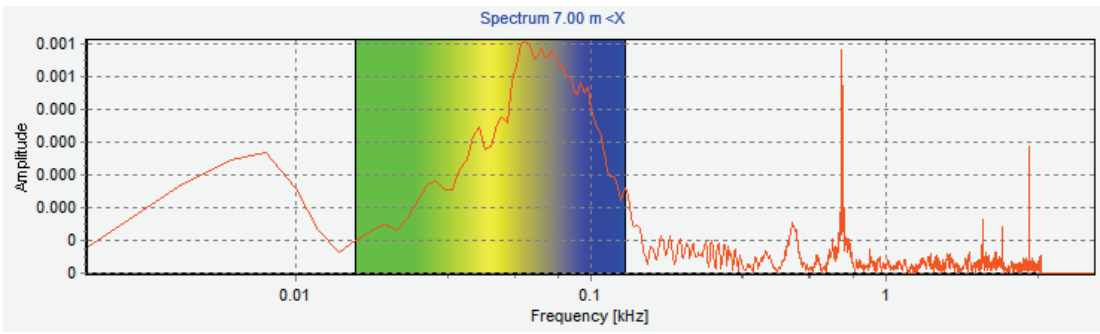

Fig. 5. Spectrum of a wave record presented in Fig. 2 and the range of frequency considered

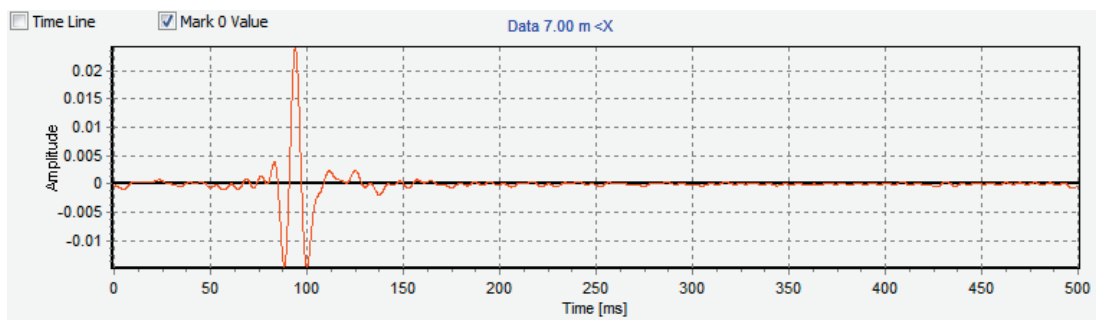

Fig. 6. A sample of registered vibration after filtering procedure 
I. BAGIŃSKA et al.
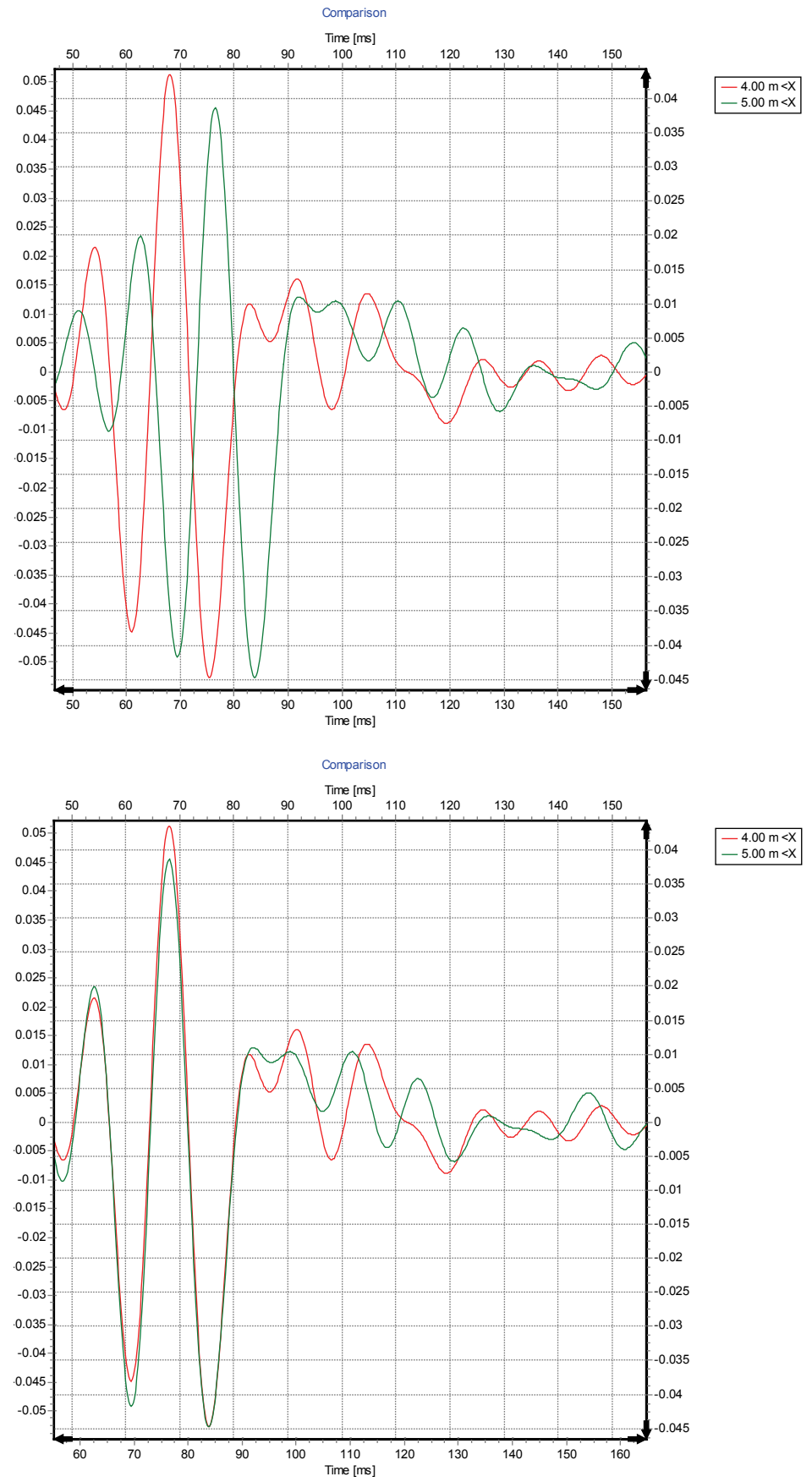

Fig. 7. Graphs of filtered wave records at two consecutive depths: before shifting at the top and shifted at the bottom 
2. If the seismic module consists of several accelerometers, then it is recommended to consider waves recorded by the same one.

3. Before the wave records from consecutive depths are compared, they are filtered with use of Fourier transform. It is admissible to use different filter settings for different depth intervals, but they need to be identical for a pair of waves being compared. Figure 5 presents the frequency spectrum of the sample result from Fig. 2 and the range of frequency to be filtered. A subsequent graph in Fig. 6 presents the filtered wave record.

4. The time difference, in which the wave reaches a given depth level is determined by overlapping the filtered record of vibration at a greater depth over the record obtained at a lower depth and shifting along the time axis. That time is automatically calculated by SEISMIC-pro software once the shifting is completed. The shift should be imposed so that the best fit is obtained (see Fig. 7).

5. Wave velocity is calculated automatically by the program. A sample report of $\mathrm{S}$-wave velocity computation is presented in Fig. 8.

Fig. 8. Graphs of filtered records of the wave at consecutive depths

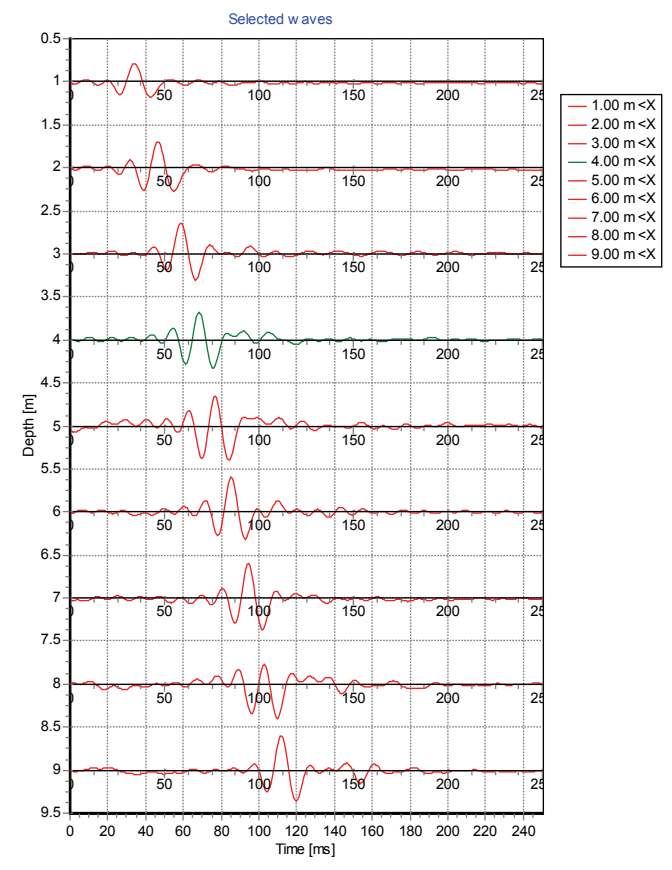

\subsection{DETERMINATION OF SHEAR MODULUS}

The value of small strain shear modulus can be calculated on the basis of shear wave velocity applying the following formula 


$$
G_{\max }=\rho\left(V_{s}\right)^{2}
$$

where: $\rho$-bulk density, $V_{s}-$ shear wave velocity.

Values of wave velocity provided by SEISMIC-pro software were exported to another program: CPT-pro. The program allows the user to calculate modulus $G_{\max }$ and also to present results of standard CPTU. Figure 9 presents basic CPTU test results, i.e., recorded values of $q_{c}, f_{s}, u_{2}$ and derived $R_{f}$ as well as values of $V_{s}$ imported

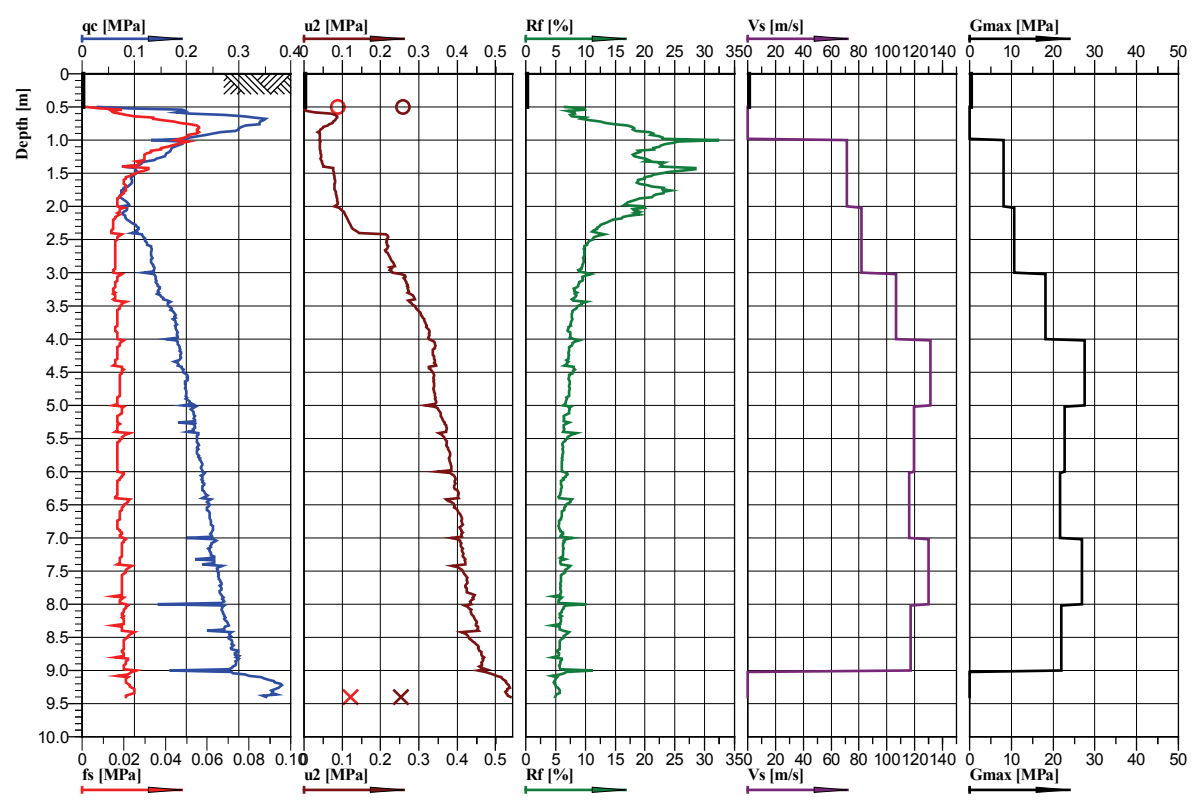

Fig. 9. Recorded values of $q_{c}, f_{s}, u_{2}$, calculated and derived $R_{f}$ and interpreted $V_{s}$ and $G_{\max }$

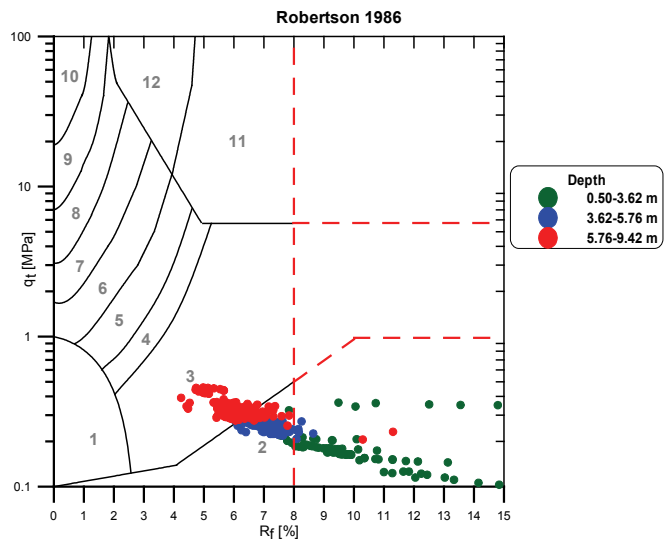

\begin{tabular}{|c|l|}
\hline SBT & \multicolumn{1}{|c|}{ Robertson 1986 } \\
\hline 1 & Sensitive fine grained \\
\hline 2 & Organic material \\
\hline 3 & Clay \\
\hline 4 & Silty clay to clay \\
\hline 5 & Clayey silt to silty clay \\
\hline 6 & Sandy silt to clayey silt \\
\hline 7 & Silty sand to sandy silt \\
\hline 8 & Sand to silty sand \\
\hline 9 & Sand \\
\hline 10 & Gravelly sand to sand \\
\hline 11 & Very stiff fine grained \\
\hline 12 & Sand to clayey sand \\
\hline
\end{tabular}

Fig. 10. Measuring points on the graph of extended classification by Robertson (1986) 
from SEISMIC-pro and $G_{\max }$ calculated in accordance with (1). All values are presented on the graph as functions of depth.

Finally, the soil type has been identified on the basis of the extended classification by Robertson (1986) [2] (see Fig. 10). The soil was determined to be clay and/or organic material. Soil consistency was determined as very soft.

\section{CONCLUSIONS}

Preciseness and correctness of a seismic test as well as a proper interpretation procedure play a key role in proper determination of shear modulus $G_{\max }$. Sources of disturbance in measurements include extrinsic noise, incorrect and not repeatable hits as well as incorrect attachment and load of an anvil. Setting a proper measuring range and instrument calibration are also very important.

The discussed method used to evaluate seismic wave velocity is precise and effective. It provides a more accurate estimation than in the cross-correlation method due to the elimination of some sources of disturbance. Use of selective filtering of the wave ensures elimination of the noise effect. Moreover, it is not necessary to perform "left" and "right" hits. A single strike at each depth is enough for the presented methodology. That may decrease the time and costs of investigations.

The in situ seismic test (SCPTU) performed simultaneously with a standard CPTU one makes it possible to determine strength as well as deformation parameters and as such it provides full recognition of the subsoil.

\section{REFERENCES}

[1] AReias L., Van IMPe W., Interpretation of SCPT Data Using Cross-over and Cross-Correlation Methods, Engineering Geology for Infrastructure Planning in Europe, Springer, 2004.

[2] BAGIŃSKA I., Analiza oceny rodzaju gruntu ustalonego na podstawie badań CPTU, Geoinżynieria, Drogi, Mosty, Tunele, 2012, nr 2, 38-45.

[3] BAJDA M., Źródło generacji fali sejsmicznej w sondowaniach SCPT, Przegląd Naukowy Wydziału Inżynierii i Kształtowania Środowiska, 2009, No. 4 (46), 57-66.

[4] FAJKLEwicz Z., Zarys geofizyki stosowanej, Wydawnictwo Geologiczne, Warszawa 1972.

[5] Lunne T., Robertson P.K., Powell J.J.M., Cone Penetration Testing in geotechnical practice, Blackie Academic and Professional, London 1997. 